\title{
Total laparoscopic en bloc right hemicolectomy and pancreaticoduodenectomy with transvaginal specimen extraction for locally advanced right colon cancer: a case report
}

\author{
Haipeng Meng ${ }^{1}$, Hui Xu ${ }^{2}$, Xiaoming Wang ${ }^{2}$, Longyi Chen ${ }^{2}$, Fan Yang ${ }^{2}$, Ruichao Geng ${ }^{2 \#}$, Yanling Xu ${ }^{3 \#}$, \\ Gang Yu ${ }^{1,2 \#}$ \\ ${ }^{1}$ Department of General Surgery, Qilu Hospital (Qingdao), Cheeloo College of Medicine, Shandong University, Qingdao, China; ${ }^{2}$ Department of \\ Colorectal Surgery, Zibo Municipal Hospital, Zibo, China; ${ }^{3}$ Department of Respiratory Medicine, Qilu Hospital (Qingdao), Cheeloo College of \\ Medicine, Shandong University, Qingdao, China \\ \#These authors contributed equally to this work. \\ Correspondence to: Gang Yu, MD, PhD. Department of General Surgery, Qilu Hospital (Qingdao), Cheeloo College of Medicine, Shandong \\ University, 758 Hefei Road, Qingdao 266035, China. Email: ygzb2020@163.com.
}

\begin{abstract}
For locally advanced right colon cancer (LARCC) invading duodenum, multivisceral resection is a curative surgical treatment, which is technically challenging when performed in a total laparoscopic approach. Herein, we report the first case of LARCC treated by total laparoscopic en bloc right hemicolectomy and pancreaticoduodenectomy with transvaginal specimen extraction. The patient was a 37-year-old female suffering from upper abdominal pain who was diagnosed with LARCC invading the duodenum by preoperative examination. The en bloc resection and digestive tract reconstruction were completed laparoscopically without an assisted abdominal incision. Then the specimen was extracted transvaginally through a $6 \mathrm{~cm}$ transverse incision made in the posterior vaginal fornix and the vaginal incision was closed by a continuous suture intracorporeally. The operative time was $470 \mathrm{~min}$ and intraoperative blood loss was $130 \mathrm{~mL}$. The postoperative pathological examination showed T4bN0M0 adenocarcinoma of the hepatic flexure of colon with infiltration of duodenal serosa, and all the margins were negative. The patient recovered uneventfully with minimal postoperative pain and was discharged from hospital on postoperative day 7. After 3 years of follow-up, the patient was alive with no recurrence. To the best of our knowledge, this is the most extensive multivisceral resection with natural orifice specimen extraction (NOSE) ever reported. We believe that NOSE surgery, with advantages of minimal invasiveness and enhanced recovery, is a feasible and promising option for LARCC.
\end{abstract}

Keywords: Colonic neoplasms; natural orifice specimen extraction (NOSE); pancreaticoduodenectomy; case report

Submitted Nov 01, 2020. Accepted for publication Jan 29, 2021.

doi: $10.21037 /$ gs-20-800

View this article at: http://dx.doi.org/10.21037/gs-20-800

\section{Introduction}

Locally advanced right colon cancer (LARCC), defined as the invasion of neighboring organs without distant metastases, accounts for $11-28 \%$ of all right colon cancer (1). For LARCC invading duodenum, there are three main methods of surgical intervention, i.e., right hemicolectomy with (I) separation of colon cancer from the duodenum, (II) partial duodenectomy and (III) en bloc pancreaticoduodenectomy. The malignant infiltration rate in adhesion between the tumor and adjacent organs in LARCC has been reported to range from $71 \%$ to $94 \%$ (2). Therefore, such kind of adhesion should be assumed to be malignant infiltration and separation may lead to tumor recurrence. Previous reports demonstrated that for 


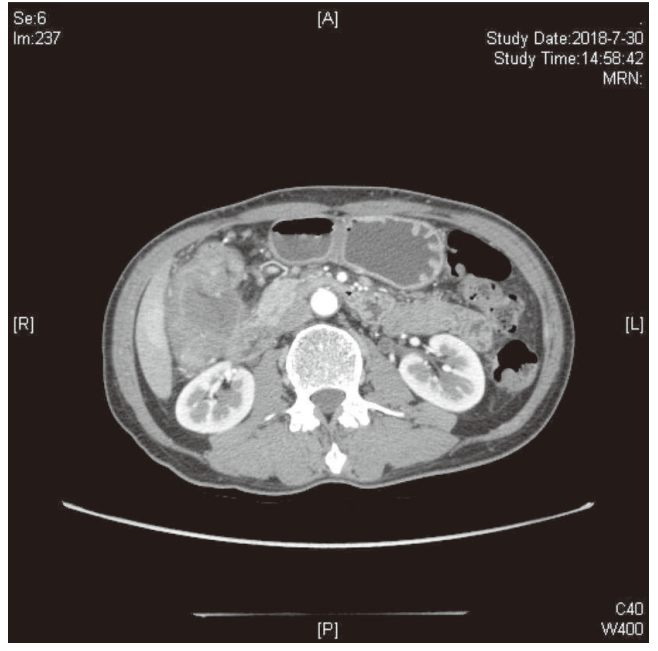

Figure 1 Contrast-enhanced computed tomography showing the mass in hepatic flexure of colon involving duodenum.

patients with LARCC invading adjacent organs, en bloc multivisceral resection led to a good prognosis, with a 21-55\% 5-year survival rate (3). In comparison, a partial duodenal wall resection was found to be associated with a poor prognosis (3). En bloc right hemicolectomy and pancreaticoduodenectomy for LARCC was first reported in 1953 (4). The majority of these procedures were carried out in an open approach, whereas Khaled et al. described the first laparoscopic hand-assistant approach (5). To the best of our knowledge, there has been no reports performing en bloc right hemicolectomy and pancreaticoduodenectomy in a total laparoscopic approach.

As an emerging minimally invasive surgical procedure, natural orifice specimen extraction (NOSE) surgery reduces surgical trauma by avoiding extraction-site laparotomy, which means avoidance of incision-related complications, mild postoperative pain, fast recovery and improved cosmetic result. It has been widely implemented and achieved satisfactory outcomes in colorectal surgery (6). But it also means the resection and reconstruction must be performed in a total laparoscopic approach, which is demanding for multivisceral resection. This report is to illustrate the detailed surgical procedure in the first case of total laparoscopic en bloc right hemicolectomy and pancreaticoduodenectomy with transvaginal specimen extraction for LARCC. We present the following case in accordance with the CARE reporting checklist (available at http://dx.doi.org/10.21037/gs-20-800).

\section{Case presentation}

A 37-year-old female patient complaining of dull upper abdominal pain for six months was admitted to the colorectal surgery department of Zibo Municipal Hospital in September 2018. She had no comorbidities and her body mass index (BMI) was $20.7(\mathrm{~kg} / \mathrm{m} 2)$. The physical examination revealed soft abdomen with upper abdominal tenderness and no rebound tenderness. The serum carcinoembryonic antigen (CEA) was $38.54 \mathrm{ng} / \mathrm{mL}$, other tumor markers and routine laboratory test results were all normal. The contrast-enhanced computed tomography revealed a $7.0 \mathrm{~cm} \times 5.0 \mathrm{~cm}$ mass in the hepatic flexure of colon with duodenum involvement (Figure 1). She was diagnosed with adenocarcinoma of the ascending colon by colonoscopic biopsy. So the preoperative diagnosis was LARCC invading the duodenum (cT4bN0M0). She had no history of abdominal surgery or significant family history. Preoperative evaluation cleared the patient from contraindications of NOSE surgery. She was fully informed of the risks and benefits of the procedure.

All procedures performed were in accordance with the ethical standards of the institutional and/or national research committee(s) and with the Helsinki Declaration (as revised in 2013). Written informed consent was obtained from the patient.

\section{Operative procedure}

The patient was placed in the functional lithotomy position after induction of general anesthesia. A Veress needle was used to create pneumoperitoneum which was maintained in $12 \mathrm{mmHg}$. The laparoscope port was created by a $10 \mathrm{~mm}$ umbilical trocar. Two $12 \mathrm{~mm}$ trocars were placed at the bilateral flanks of rectus abdominis, respectively. One $12 \mathrm{~mm}$ trocar and one $5 \mathrm{~mm}$ trocar were placed at the bilateral sides of upper abdomen, respectively. Exploration of the whole abdominal cavity revealed that the tumor located in the hepatic flexure of colon invading duodenum with no distant metastasis.

First, the median side of the right mesocolon was incised starting from the root of ileocolic pedicle. Then the peritoneum was incised over to the superior mesenteric vessels. The ileocolic vessels were mobilized and the peritoneal window was extended from middle to lateral. The origins of the ileocolic vessels were identified from the superior mesenteric vessels, clipped and divided. Careful 


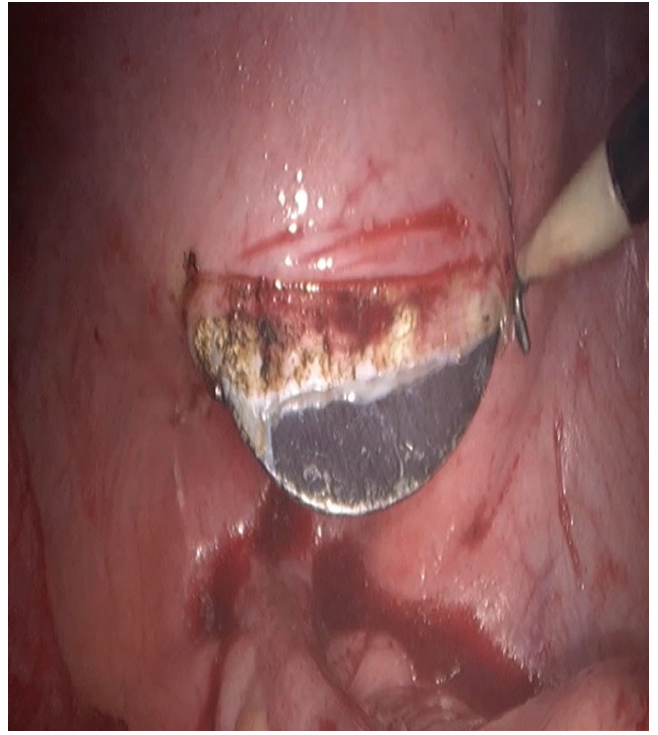

Figure 2 An incision was made on the posterior vaginal fornix.

dissection onto the duodenum and the caudal portion of the pancreas was exercised to expose the right colic vessels. Since en bloc multivisceral resection was planned, the Henle's trunk was ligated and divided at its root instead of dissecting the right colic vein, right gastroepiploic vein and anterior superior pancreaticoduodenal vein separately as was done in conventional right hemicolectomy. Dissection of the ventral side of the superior mesenteric vein permitted a complete dissection of the root of the middle colic vessels. The middle colic artery and vein were ligated separately at the lower border of pancreas. All the vessels supplying the right colon were ligated up to this point.

The ileocecal region was mobilized from medial to lateral. The mobilization was adequate to facilitate anastomosis under laparoscopy. The mesentery was incised up to the bowel edge, and the vessels were ligated along the dissection. The ileum was transected by an endoscopic linear stapler. The greater omentum was dissected toward the edge of the transverse colon. Because en bloc resection was intended, the gastrocolic ligament was separated along the inner margin of right gastroepiploic vein rather than dissecting along the outer edge of right gastroepiploic vein, as was done in conventional right hemicolectomy. Likewise, the gastroduodenal artery was ligated and divided at its root, with no need to ligate the right gastroepiploic artery specifically. The stomach was transected via an endoscopic linear stapler. The transverse mesocolon was dissected and the transverse colon division was performed using a $60 \mathrm{~mm}$ endoscopic linear stapler.

As the right colon was mobilized completely, the operative field was broad when it came to the duodenum mobilization. The ligament of Treitz was mobilized and the jejunum was dissected via endoscopic linear stapler. Division of the pancreatic neck was made by harmonic scalpel up to the region of the pancreatic duct. Once clearly identified, the pancreatic duct was divided with scissors. The common bile duct was transected with scissors following cholecystectomy. In order to prevent bile spillage, the proximal stump of the common hepatic duct was clipped by an endoscopic bull-dog clips. Up to this point, the extensive resection was completed, and the specimen was placed at the pelvic cavity within a protective bag for retrieval.

Digestive tract reconstruction was implemented in accordance with the Child's method, in the sequence of duct-to-mucosa pancreaticojejunostomy, end-to-side choledochojejunostomy and gastrojejunostomy. Then, a side-to-side anastomosis between small bowel and the transverse colon was fashioned using a stapled technique. All the anastomoses were carried out intracorporeally.

The specimen extraction procedure was as follows. The patient was tilted into head down position to expose the pelvic operative field completely. The vagina was disinfected with iodophor gauze thoroughly and the pelvic cavity was irrigated repeatedly with iodophor diluent and saline. An abdominal spatula was placed into the vagina with its tip supporting the posterior vaginal fornix, on which a $6 \mathrm{~cm}$ transverse incision was made by electrocautery (Figure 2). A double-ringed wound protective device was used to protect the vaginal incision. The specimen was grasped with an ovary clamp and pulled into the vagina through the vaginal incision. Then it was removed through the vagina in a snakelike fashion (Figure 3), carefully maintaining its integrity as well as the pneumoperitoneum. After removal of the specimen and the wound protector, the vaginal incision was closed with a 3-0 V-LOC suture intracorporeally (Figure 4). A drainage tube was placed in the right side of the abdominal cavity via the $5 \mathrm{~mm}$ port.

\section{Intraoperative and postoperative outcomes}

The operative time was $470 \mathrm{~min}$, in which the duration of specimen extraction was $31 \mathrm{~min}$. Intraoperative blood loss was $130 \mathrm{~mL}$ with no blood transfused. The diameter of specimen was $7 \mathrm{~cm}$ (Figure 5). The number of lymph nodes harvested was 28 . The pathological examination (hematoxylin-eosin staining) showed pT4bN0M0 ulcerative 

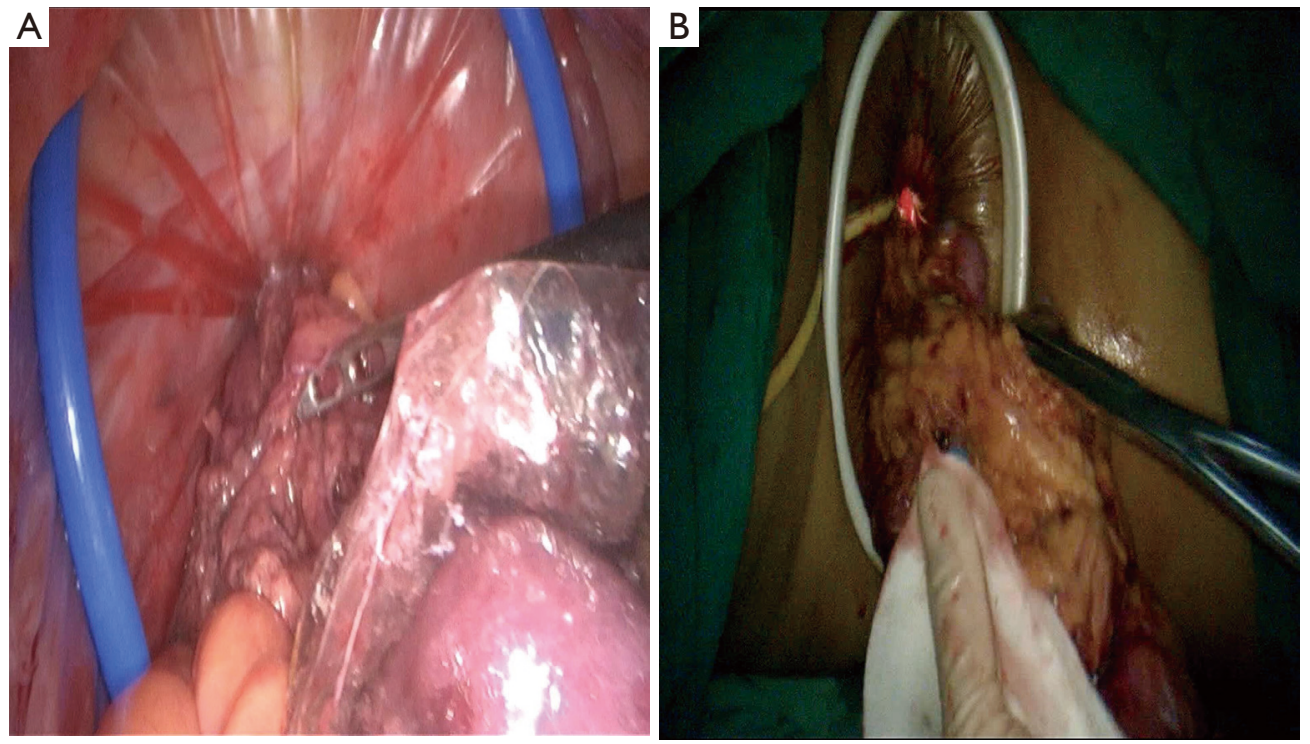

Figure 3 Transvaginal specimen extraction. (A) Intracorporeal view. (B) Extracorporeal view.

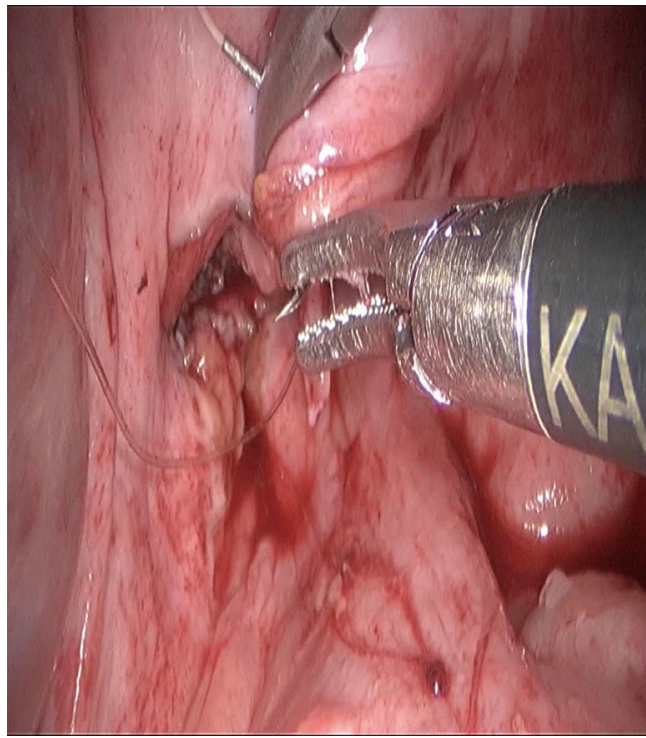

Figure 4 The vaginal incision was closed by a continuous suture.

adenocarcinoma of the hepatic flexure of colon infiltrating the serosa of duodenum (Figure 6), and all the margins were negative. The postoperative pain was mild with no opioids required. The patient started mobilization the day after surgery and daily diet was initiated on post operative day 5. She recovered uneventfully and was discharged on postoperative day 7 . The adjuvant chemotherapy was administered as routine. After 3 years of follow-up, the

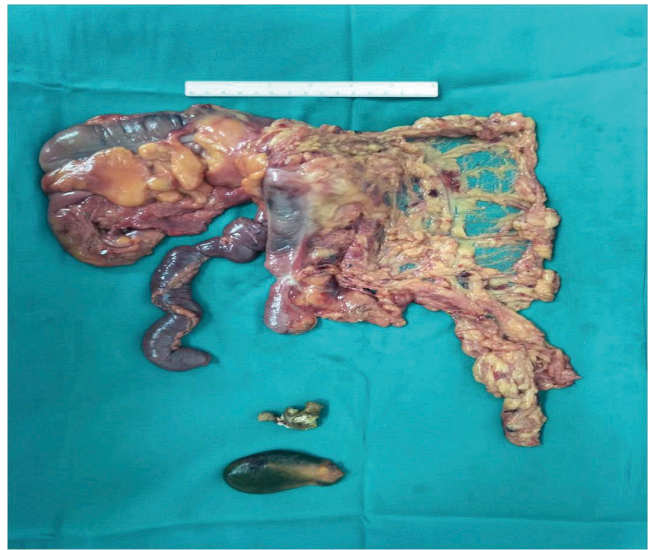

Figure 5 Gross appearance of resected specimen.

patient was alive with no recurrence.

\section{Discussion}

Since July 2018, we have performed five cases of laparoscopic NOSE pancreaticoduodenectomy including the present one. According to our experience, vagina is the optimal access route for specimen extraction for female patients. Because it is wider, more elastic and associated with relatively low morbidity compared with rectum. In fact, transvaginal specimen extraction has been frequently reported in NOSE surgery (6). 


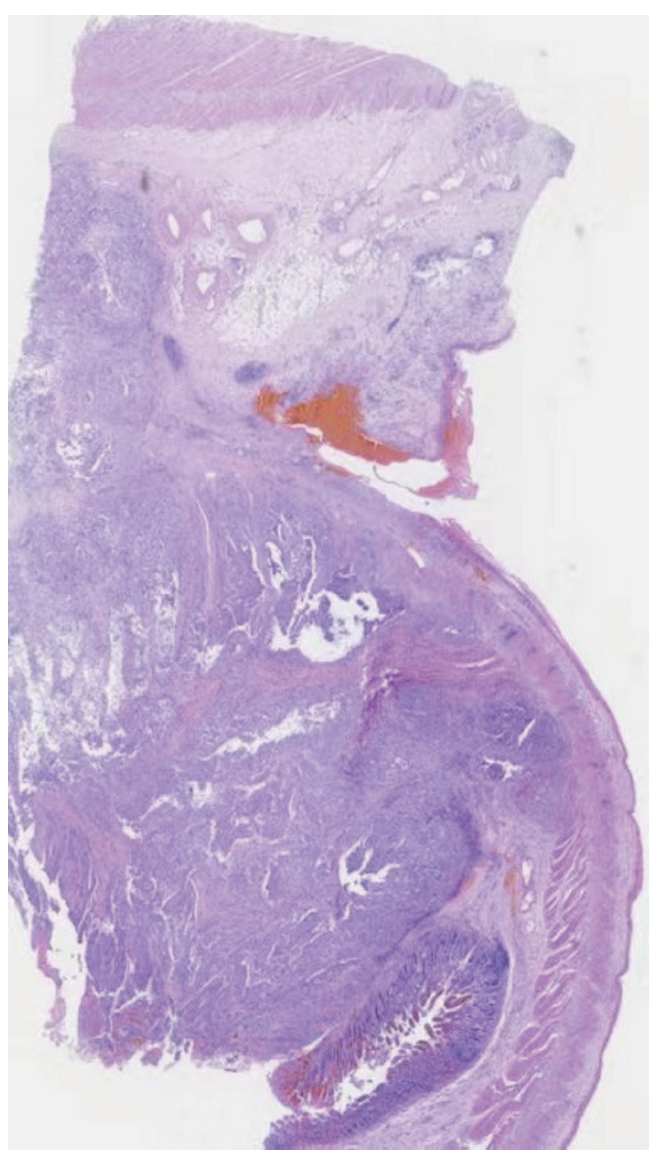

Figure 6 Hematoxylin-eosin staining $(\times 100)$ showing colonic cancer infiltrating serosa of duodenum.

In the literature, rectovaginal fistula, pelvic abscess and bladder dysfunction have been reported as major complications due to use of the transvaginal route (7). Gündoğan $e t a l$. even reported a case of vaginal recurrence of colon cancer following transvaginal tumor extraction without vaginal incision protection (8). We suggest the following measures be taken to prevent those complications. First, the specimen should be placed in a specimen bag once resected. Second, the vagina must be disinfected with iodophor gauze thoroughly before being cut open. Third, the posterior vaginal fornix should be supported by an abdominal spatula before being incised in order to prevent damages to bladder or rectum. Fourth, a wound protector is recommended to protect the vagina during specimen extraction. Fifth, the pelvic cavity ought to be irrigated extensively with iodophor diluent and saline after closure of the vaginal incision.

The abdominal wall is subjected to innervation of somatic nerves and sensitive to painful stimuli, but internal organs such as the vagina are not. Because of absence of abdominal auxillary incision, the incision-related complications are prevented and the postoperative pain is mild, which we believe would enhance the postoperative recovery. Of note, the young female patient was especially satisfied with the cosmetic effect of the procedure.

In conclusion, NOSE surgery is a feasible and promising option for LARCC which deserves further exploration. However, we believe that it is a challenging procedure, highlighting that expertise in minimally invasive colorectal surgery and NOSE must be a prerequisite for surgeons aspiring to reproduce our result.

\section{Acknowledgments}

We thank Yeqin Hu for editing the English text of a draft of this manuscript.

Funding: None.

\section{Footnote}

Reporting Checklist: The authors have completed the CARE reporting checklist. Available at http://dx.doi.org/10.21037/ gs-20-800

Conflicts of Interest: All authors have completed the ICMJE uniform disclosure form (available at http://dx.doi. org/10.21037/gs-20-800). The authors have no conflicts of interest to declare.

Ethical Statement: The authors are accountable for all aspects of the work in ensuring that questions related to the accuracy or integrity of any part of the work are appropriately investigated and resolved. All procedures performed in studies involving human participants were in accordance with the ethical standards of the institutional and/or national research committee(s) and with the Helsinki Declaration (as revised in 2013). Written informed consent was obtained from the patient.

Open Access Statement: This is an Open Access article distributed in accordance with the Creative Commons Attribution-NonCommercial-NoDerivs 4.0 International License (CC BY-NC-ND 4.0), which permits the noncommercial replication and distribution of the article with the strict proviso that no changes or edits are made and the original work is properly cited (including links to both the 
formal publication through the relevant DOI and the license). See: https://creativecommons.org/licenses/by-nc-nd/4.0/.

\section{References}

1. Fuks D, Pessaux P, Tuech JJ, et al. Management of patients with carcinoma of the right colon invading the duodenum or pancreatic head. Int J Colorectal Dis 2008;23:477-81.

2. Sheng QS, Chen WB, Li MJ, et al. Combined right hemicolectomy and pancreaticoduodenectomy for locally advanced right hemicolon cancer. Hepatobiliary Pancreat Dis Int 2015;14:320-4.

3. Saiura A, Yamamoto J, Ueno M, et al. Long-term survival in patients with locally advanced colon cancer after en bloc pancreaticoduodenectomy and colectomy. Dis Colon Rectum 2008;51:1548-51.

4. Van Prohaska J, Govostis MC, Wasick M. Multiple organ

Cite this article as: Meng H, Xu H, Wang X, Chen L, Yang F, Geng R, Xu Y, Yu G. Total laparoscopic en bloc right hemicolectomy and pancreaticoduodenectomy with transvaginal specimen extraction for locally advanced right colon cancer: a case report. Gland Surg 2021;10(5):1780-1785. doi: 10.21037/gs20-800 resection for advanced carcinoma of the colon and rectum. Surg Gynecol Obstet 1953;97:177-82.

5. Khaled YS, Ammori MB, Sharif HI, et al. Simultaneous laparoscopic subtotal colectomy and pancreaticoduodenectomy for colonic FAP and ampullary cancer. Surg Laparosc Endosc Percutan Tech 2012;22:e79-82.

6. Guan X, Liu Z, Longo A, et al. International consensus on natural orifice specimen extraction surgery (NOSES) for colorectal cancer. Gastroenterol Rep (Oxf) 2019;7:24-31.

7. Ghezzi F, Cromi A, Ciravolo G, et al. A new laparoscopictransvaginal technique for rectosigmoid resection in patients with endometriosis. Fertil Steril 2008;90:1964-8.

8. Gündoğan E, Cicek E, Sumer F, et al. A case of vaginal recurrence following laparoscopic left-sided colon cancer resection combined with transvaginal specimen extraction. J Minim Access Surg 2019;15:345-7. 